\title{
Espinha Bifida Aberta: Achados Ultra-sonográficos e Presença de Contrações Uterinas na Predição da Evolução Motora Neonatal
}

\author{
Open Spina Bifida: Antenatal Ultrasound Findings and Uterine Contractions as \\ Predictors of the Neonatal Neuromotor Outcome
}

\begin{abstract}
Wagner Jou Hisaba, Antônio Fernandes Moron, Sérgio Cavalheiro, Renato Martins Santana, Jurandir Piassi Passos, Eduardo Cordioli
\end{abstract}

\begin{abstract}
RESUM0
Objetivo: avaliar a influência das alterações ultra-sonográficas pré-natais e das contrações uterinas de trabalho de parto na evolução motora neonatal em fetos portadores de espinha bífida aberta.

Métodos: foram analisados fetos portadores de espinha bifida aberta. Estes fetos foram acompanhados nos serviços de Medicina Fetal do Hospital São Paulo (Universidade Federal de São Paulo) e do Hospital e Maternidade Santa Joana. Todos os partos foram realizados nestes serviços e a avaliação neonatal foi realizada pela equipe de Neurocirurgia comum a ambas as instituições Foi observada a influência das alterações ultra-sonográficas (macrocrania, microcrania, nível da falha de fechamento da coluna, pé torto e tipo de apresentação fetal) na força muscular de membros inferiores no período neonatal. Foi analisada, também, a influência das contrações uterinas sobre a movimentação dos membros inferiores. Todos os partos foram realizados por cesárea. Foram utilizados os testes de $\chi^{2}$ e Fisher para comparações categóricas, com $p<0,05$ para estabelecer associação significante.

Resultados: foram estudados cinqüenta e três casos de espinha bífida isolada. As alterações da circunferência craniana e o nível da lesão não interferiram na parte neuromotora neonatal. Entretanto, a presença de pé torto e a apresentação pélvica mostraram prognóstico neurológico neonatal desfavorável $(p<0,05)$. O pé torto foi encontrado em 23 fetos (43\%). Dezoito recém-nascidos $(78,3 \%)$ portadores de pé torto apresentaram alterações motoras. Nenhum neonato em apresentação pélvica $(n=10)$ mostrou função motora normal. A diminuição da força motora nos membros inferiores também foi observada em 13 fetos (87\%) expostos às contrações uterinas de trabalho de parto e à rotura prematura de membranas $(p<0,05)$.

Conclusão: a presença de pé torto e a apresentação pélvica são parâmetros úteis para a predição da evolução neurológica neonatal. Contrações uterinas de trabalho de parto e a rotura prematura de membrana são fatores de pior prognóstico neuromotor neonatal.
\end{abstract}

PALAVRAS-CHAVE: Espinha bífida aberta. Alteração motora, neonatal. Ultra-sonografia pré-natal.

Disciplina de Medicina Fetal da Universidade Federal de São Paulo, Disciplina de Neurocirurgia da Universidade Federal de São Paulo

Correspondência:

Wagner Jou Hisaba

Rua Dom Duarte Leopoldo, 932

01542-000 - São Paulo - SP

Fone: (11) 3208-0681 / Fax (11) 5572-4449

e-mail: hisaba@uol.com.br
Introdução

A espinha bífida é uma das mais freqüentes malformações congênitas no mundo e é responsável por importantes seqüelas neurológicas. A incidência desta malformação é de 0,2 a 0,4 por mil nascidos vivos, com diminuição destes valo- 
res desde a década de 70 , quando atingiam 0,5 a 0,6 por mil nascidos vivos ${ }^{1}$. Esta diminuição de sua incidência deveu-se aos programas de orientação sobre o uso de ácido fólico na prevenção dos defeitos do tubo neural e ao aperfeiçoamento do diagnóstico pré-natal por meio da ultra-sonografia, permitindo a interrupção eletiva da gestação em países onde a legislação permite tal prática.

A sensibilidade da ultra-sonografia para a detecção da mielomeningocele é de $80-90 \%{ }^{2,3}$. Esta sensibilidade depende da qualidade profissional do examinador e dos equipamentos utilizados na avaliação fetal. Profissionais bem treinados podem identificar o nível e a extensão da abertura da coluna vertebral. Kollias et al. ${ }^{4}$ relataram concordância da localização da espinha bífida aberta entre a ultra-sonografia pré-natal e a avaliação pós-natal em $64 \%$ dos casos e com discordância de apenas um segmento vertebral em $79 \%$.

As alterações motoras e sensitivas variam conforme o nível da lesão e o grau de comprometimento da medula. A necessidade de cadeira de rodas para locomoção ocorre em 90, 45 e 17\% dos pacientes com lesão em nível tóraco-lombar, lombar e sacral, respectivamente. A deambulação sem nenhum apoio é possível em $57 \%$ dos indivíduos com lesão sacral, $7 \%$ em indivíduos com lesão lombar e nenhum com lesão tóraco-lombar ${ }^{5}$.

Há poucos trabalhos que associaram os achados ultra-sonográficos pré-natais ao prognóstico neuromotor pós-natal. Em estudo envolvendo 26 pacientes, Brumfield et al. ${ }^{6}$ demonstraram que a macrocefalia (diâmetro biparietal e circunferência craniana maiores que o percentil 95 para a idade gestacional) estava associada a péssimo prognóstico. Biggio et al. ${ }^{7}$ avaliaram 33 casos de espinha bífida isolada com diagnóstico pré-natal. Observaram que a presença de dilatação dos ventrículos cerebrais e a presença de pé torto não alteraram a capacidade motora destes pacientes.

Durante as três últimas décadas, muito se discutiu sobre a melhor via de parto para os fetos portadores de espinha bifida aberta. Luthy et al. ${ }^{8}$ demonstraram melhor função motora em crianças nascidas por cesárea antes do trabalho de parto quando comparadas às crianças nascidas por via vaginal ou por cesárea após a instalação das contrações uterinas. A presença de rotura de membranas com ou sem contrações uterinas subseqüentes também foi relacionada a prognóstico motor menos satisfatório ${ }^{9}$.

Procuramos, por meio deste trabalho, analisar a associação entre a evolução neuromotora neonatal e os parâmetros ultra-sonográficos da espinha bífida pré-natal como nível da espinha bífida, valor da circunferência craniana, pé torto e apresentação fetal. Procuramos, também, avaliar a interferência das contrações de trabalho de parto e rotura prematura de membranas na evolução neurológica neonatal.

\section{Pacientes e Métodos}

Este é um estudo retrospectivo que incluiu gestantes cujos fetos eram portadores de espinha bífida aberta e que foram acompanhadas nos serviços de Medicina Fetal do Hospital São Paulo da Universidade Federal de São Paulo (Escola Paulista de Medicina) e do Hospital e Maternidade Santa Joana, no período de janeiro de 1993 a dezembro de 2001 . Seus partos foram realizados nestes hospitais. Os critérios de exclusão incluíam: recémnascidos portadores de espinha bífida aberta sem diagnóstico pré-natal ou com diagnóstico pré-natal mas sem avaliação ultra-sonográfica dos setores de Medicina Fetal das instituições envolvidas no estudo e presença de alterações cromossômicas e outras malformações associadas. Excluímos, também, dois casos que foram submetidos à derivação céfalo-amniótica.

Todos os exames ultra-sonográficos pré-natais foram realizados por, no mínimo, dois ultrasonografistas vinculados aos serviços de Medicina Fetal daquelas instituições. A avaliação ecográfica foi realizada mediante o uso de aparelho Shimadzu 500 C, Diasonic Synergy e GE Logic 500 com transdutor de 3,5 MHz.

O nível da lesão foi classificado em sacral, lombo-sacral, lombar e tóraco-lombar. O tipo de lesão foi classificado em meningocele (defeito de fechamento ósseo da coluna com protrusão somente das meninges e líquido cérebro-espinhal) e meningomielocele (saída de medula espinhal ou outras estruturas no interior do saco herniário). A dilatação do ventrículo foi caracterizada com a medida do átrio maior ou igual a $10 \mathrm{~mm}$. Presença de microcefalia ou macrocefalia foi definida quando a circunferência craniana estava abaixo do percentil 3 e acima do percentil 97 , respectivamente, para a idade gestacional. Pé torto foi identificado conforme o alinhamento dos pés em relação à tíbia e à fíbula.

Foi analisada a presença de contrações uterinas de trabalho de parto antes do nascimento e o tipo de apresentação fetal.

A avaliação da evolução neonatal foi realizada pela equipe de Neonatologia e pela equipe de Neurocirurgia do Hospital São Paulo e do Hospital e Maternidade Santa Joana. Todos os profissionais envolvidos atuam em ambos os serviços. O nível de lesão foi avaliado clinicamente por radiografia e durante o ato operatório para a sua correção. A 
presença de dilatação dos ventrículos laterais foi confirmada pela avaliação ultra-sonográfica ou por tomografia computadorizada. A avaliação clínica da força motora foi realizada por neurologistas pediátricos. A força motora foi classificada como alterada quando havia a presença de plegia ou paresia de um ou de ambos os membros inferiores. Quando o exame neurológico não mostrava sinais de alteração motora, foi classificada como normal. Os resultados foram analisados através de testes de $\chi^{2}$ e teste exato de Fisher, mantendo-se o nivel de significância em p<0,05 (nivel de rejeição de hipótese de nulidade).

\section{Resultados}

Foram acompanhados 33 fetos portadores de espinha bífida aberta no Hospital São Paulo UNIFESP e 20 fetos no Hospital e Maternidade Santa Joana, totalizando 53 casos.

A idade gestacional média, na ocasião do parto, foi de 37,5 semanas, com mínimo de 33 semanas e máximo de 41 semanas e desvio-padrão de 0,230. O peso médio dos recém-nascidos na ocasião do parto foi de 3.027 gramas, com desvio- padrão de 86,5 gramas. A adequação ao peso gestacional esteve presente em 50 casos $(94,3 \%)$.

Foram observados 7 casos de abertura em nível sacral, 27 em nivel lombo-sacral, 14 em nível lombar e 4 em região tóraco-lombar. A dilatação dos ventrículos laterais foi encontrada em 52 casos. Em apenas um caso, a ventriculomegalia não foi identificada durante o período pré-natal e neonatal; neste caso, a alteração encontrava-se em nivel sacral. Houve 52 casos de mielomeningocele. Houve apenas um caso de meningocele em região sacral e sem ventriculomegalia.

Em nove casos (17\%) não houve concordância entre o nível de lesão observado durante o exame pré-natal e o nível identificado durante o período neonatal.

Não houve diferença estatisticamente significativa entre o nível de lesão e a alteração motora do recém-nascido (Tabela 1). A presença de alterações no tamanho da circunferência craniana observada nos exames pré-natais não apresentou influência sobre a parte motora neonatal (Tabela 2). A presença de pé torto, entretanto, apresentou associação significativa com a função motora (Tabela 2). Dezoito fetos (78,3\%) portadores de pé torto apresentaram alteração neuromotora.

Tabela 1 - Distribuição dos indivíduos quanto à condição da força motora nos membros inferiores e ao nível da lesão vertebral.

\begin{tabular}{|c|c|c|c|c|c|}
\hline \multirow[b]{2}{*}{ Força motora } & \multicolumn{5}{|c|}{ Nível da lesão } \\
\hline & Sacral & Lombo-sacral & Lombar & Tóraco-lombar & Total \\
\hline Normal & $5(71,4 \%)$ & $8(29,6 \%)$ & $7(50 \%)$ & $0(0 \%)$ & $20(37,7 \%)$ \\
\hline Alterada & $2(28,6 \%)$ & $19(70,4 \%)$ & $7(50 \%)$ & $5(100 \%)$ & $33(62,3 \%)$ \\
\hline Total & 7 & 27 & 14 & 5 & 53 \\
\hline
\end{tabular}

Tabela 2 - Influência dos parâmetros ultra-sonográficos e das contrações uterinas na evolução motora neonatal.

\begin{tabular}{llcc}
\hline & \multicolumn{2}{c}{ Força motora } & \\
& Alterada & Normal & p \\
\hline Macro/microcrania & $16(76 \%)$ & $5(24 \%)$ & 0,129 \\
Pé torto & $23(53,5 \%)$ & $30(46,5 \%)$ & 0,035 \\
Apresentação pélvica & $10(100 \%)$ & $0(0 \%)$ & 0,008 \\
Contrações uterinas & $13(86,7 \%)$ & $2(13,3 \%)$ & 0,009 \\
\hline
\end{tabular}

Todos os partos foram realizados por cesárea com indicação, em todos os casos, pela presença da espinha bífida aberta.

No momento do parto, o tipo de apresentação e a presença de contrações uterinas influenciaram a evolução motora neonatal. Dez fetos encontravam-se em apresentação pélvica. Nenhum deles apresentou padrão normal de força motora (Tabela 2). As gestantes portadoras destes fetos não apresentaram contrações uterinas ou amniorrexe antes do parto. Na distribuição do nível de lesão, foram observados dois casos de lesão torácica, dois em topografia lombar, quatro em nível lombo-sacral e dois em região sacral.

Quarenta e três fetos encontravam-se em apresentação cefálica. Cinqüenta e três por cento destes fetos apresentaram alteração da motricidade de membros inferiores.

A presença de contrações uterinas seguidas ou não de rotura espontânea das membranas mostrou diminuição da força motora em membros inferiores estatisticamente significativa. Nestes fetos, foi observada a seguinte distribuição das lesões: 2 torácicas, 2 lombares, 4 lombosacrais e 2 sacrais. 


\section{Discussão}

Durante 20 anos, autores procuraram associar as alterações ultra-sonográficas fetais com a evolução neuromotora após o parto. A altura da lesão vertebral, a presença de ventriculomegalia associada à macrocefalia e a presença de pé torto congênito foram os principais marcadores pesquisados para estabelecer esta associação $0^{6,7,10}$.

A ventriculomegalia ou dilatação do sistema dos ventrículos laterais do cérebro fetal ocorre devido à tração do tronco cerebral e cerebelo pelo forame magno, promovendo obstrução ao fluxo do líquor através dos forames de Luschka e Magendie. Esta obstrução ocorre em 45 a $86 \%$ dos casos de espinha bífida aberta ${ }^{11}$. Em nossa casuística, a ausência de dilatação dos ventrículos laterais ocorreu em apenas um caso, de localização sacral.

A ultra-sonografia antenatal permite a identificação do nível de abertura da coluna vertebral, por meio de pontos de referência do feto. A identificação precisa da topografia da lesão depende de uma série de fatores que incluem a posição fetal, a qualidade do aparelho e a experiência do examinador que, em nosso trabalho, permitiram a identificação do local da falha de fechamento vertebral em $87 \%$ dos casos.

A topografia da lesão reveste-se de importância, pois está relacionada ao prognóstico neurológico. Quanto mais elevado o nível, pior é a motricidade dos membros inferiores. Noventa por cento dos indivíduos portadores de lesões em nível torácico não apresentam movimentação satisfatória dos membros inferiores, necessitando de recursos especiais para sua locomoção ${ }^{5,7}$. As repercussões neuromotoras são menos pronunciadas nas lesões sacrais, com as quais apenas $17 \%$ dos pacientes necessitam de cadeiras de rodas. Todos os recém-nascidos com lesão torácica, em nossa casuística, apresentaram comprometimento neuromotor; entretanto, analisando todos os demais segmentos vertebrais, não encontramos associação significativa com a função neurológica. Esta divergência em relação aos outros trabalhos ocorreu, provavelmente, em razão do pequeno tamanho amostral envolvido no estudo, com avaliação de apenas cinco casos de espinha bífida de localização torácica e somente sete casos de localização sacral.

A expansão do sistema liquórico com formação de macrocefalia foi relacionada a evolução motora insatisfatória, devido ao processo de hipertensão intracraniana com conseqüente lesão dos centros motores centrais ${ }^{6}$. A microcefalia, outro achado comum na espinha bífida aberta na avaliação pré-natal (62 a 69\% dos casos) ${ }^{12}$, não apresen- ta mecanismo etiológico conhecido e não há referências sobre sua influência nas funções neuromotoras. Estas alterações da circunferência craniana não representaram, em nosso trabalho, marcador ultra-sonográfico antenatal de mau prognóstico quanto à movimentação dos membros inferiores.

O pé torto congênito é achado freqüente em fetos portadores de espinha aberta. Observamos a associação entre o comprometimento motor e a presença de pé torto congênito, independente do nivel da lesão. Algumas fibras nervosas destinadas aos pés devem, de alguma forma, influenciar a inervação de partes mais superiores e, deste modo, repercutir na força muscular ${ }^{7}$.

As apresentações anômalas são freqüentes em fetos portadores de mielomeningocele (18 a $24 \%$ dos casos) quando comparados à população normal (3 a $5 \%)^{13}$, pois a hidrocefalia com aumento da circunferência craniana impede a versão interna espontânea destes fetos. Ressaltamos, em nosso trabalho, a apresentação pélvica como marcador prognóstico neurológico neonatal. Todos os fetos em apresentação pélvica mostraram comprometimento da força muscular dos membros inferiores. É importante ressaltar que estas gestantes não apresentaram contrações uterinas de trabalho de parto ou rotura espontânea das membranas antes da realização do parto. Todos apresentaram dilatação dos ventrículos laterais. Danos neurológicos foram observados nos fetos em apresentação pélvica ${ }^{5}$, quando submetidos ao parto normal, em relação ao grupo de fetos em apresentação cefálica que nasceram pela via vaginal e ao grupo de fetos que nasceram por cesárea. Alterações macroscópicas e microscópicas foram encontradas em fetos submetidos a parto normal e em apresentação pélvica ${ }^{14}$. Ocorrem danos musculares, principalmente na junção musculotendinosa caracterizados pela presença de hemorragias no perimísio com evolução para infartos musculares, devido à compressão das fibras musculares pelos hematomas. A necrose do músculo ocorre principalmente em áreas de ausência de inervação e de atrofia muscular. Hemorragias endoneurais e atrofias das fibras musculares ocorrem principalmente no nervo ciático.

O espaço diminuído na região do segmento inferior pode levar à compressão da mielomeningocele e dos músculos dos membros inferiores. Esta compressão pode restringir a movimentação dos membros inferiores e provocar conseqüente atrofia de sua musculatura. A atrofia aumenta a sensibilidade das fibras musculares aos efeitos isquêmicos e hemorrágicos que podem surgir pelo trauma das contrações de Braxton-Hicks ou pela movimentação materna. 
O parto em casos de espinha bífida fetal é controverso. Não existem estudos prospectivos e randomizados sobre a melhor via de parto neste tipo de malformação congênita. As comissões de ética dos departamentos de Perinatologia no Primeiro Mundo não permitem a realização de trabalhos neste modelo de estudo.

Foram estudadas as relações entre as contrações uterinas de trabalho de parto e a motricidade pós-natal. Em estudo retrospectivo envolvendo 160 pacientes com mielomeningocele ${ }^{8}$, crianças nascidas de cesárea sem a presença de contrações uterinas, apresentaram melhor evolução motora quando comparadas ao grupo de crianças submetidas ao parto normal ou à cesárea, mas com presença de contrações uterinas. Shurtleff et al. ${ }^{9}$ pesquisaram a evolução motora em dois grupos: com ou sem rotura de membranas. Pacientes submetidas à cesárea com membranas íntegras e em trabalho de parto apresentaram menor deterioração da força motora em comparação com o grupo com rotura de membranas e trabalho de parto submetido também à cesárea .

Nossos resultados concordam com os dados da literatura, ressaltando que a presença das contrações uterinas ou a diminuição do espaço intrauterino podem levar a alterações funcionais nos músculos e nervos dos membros inferiores. Desta forma, acreditamos que a cesárea eletiva apresenta-se como a melhor via de parto para evitar danos neurológicos a estes pacientes.

A espinha bífida aberta representa, ainda, grande dilema médico. É malformação congênita freqüente, de caráter não letal, mas com seqüelas importantes para o paciente, seus familiares e para a sociedade que cobrirá as despesas do tratamento. Seu diagnóstico pré-natal é possivel pela avaliação ultra-sonográfica. As alterações fetais encontradas, entretanto, não permitem definição quanto ao seu futuro neurológico.

Esta dúvida deixará de existir em um futuro próximo, quando a intervenção sobre a espinha bífida ocorrer, não após o nascimento, mas no interior do útero. As perspectivas da cirurgia fetal melhoram a cada dia devido ao aperfeiçoamento da tecnologia e, principalmente, devido ao empenho daqueles que acreditam em resposta terapêutica para estes pacientes.

\section{ABSTRACT}

Purpose: to determine whether prenatal sonographic findings and uterine contractions can predict neonatal motor outcome in fetuses with open spina bifida.

Methods: we evaluated retrospectively 53 fetuses with open spina bifida from 1993 to 2001. These fetuses were born and followed-up at the fetal medicine units of the Hospital São Paulo ("Universidade Federal de São Paulo") and the "Hospital Santa Joana". The influence of the alterations observed through ultrasound scan on neonatal muscular strength (macrocrania, microcrania, level of the spinal column opening, clubfoot and type of fetus position) was evaluated. The influence of uterine contractions on neonatal motor outcome was also evaluated. All deliveries were made through cesarian section. $\chi^{2}$ and Fisher tests were used for categorical comparisons. A $p<0.05$ was considered significant.

Results: Fifty-three cases of isolated open spina bifida were studied. Alterations of the cranial circumference size and the level of the lesion did not interfere in the neonatal motor perfomance. However, clubfoot and breech position showed to be predictors of an unfavorable neurological prognosis ( $p<0.05)$. Twenty-tree (43\%) infants had clubfoot. Eighteen (78.3\%) of these had abnormal leg movements. No infant in breech presentation $(n=10)$ showed normal motor function. The presence of uterine contractions and the premature membrane rupture, observed in 13 fetuses (87\%), were also linked to the alteration of neonatal muscular strength $(p<0.05)$.

Conclusion: clubfoot and breech presentation are ultrasonographic findings useful in predicting neurological neonatal outcome. Uterine contractions and premature membrane rupture are associated with poor motor neonatal outcome.

KEYWORDS: Open spina bífida. Motor outcome. Neonatal motor alterations. Antenatal ultrasonography.

\section{Referências}

1. Yen IH, Khoury MJ, Erickson JD, James LM, Waters GD, Berry RJ. The changing epidemiology of neural tube defects. United States, 1968-1989. Am J Dis Child 1992; 146:857-61.

2. Nadel AS, Green JK, Holmes LB, Frigoletto FD Jr, Benacerraf BR. Absence of need for amniocentesis in patients with elevated levels of maternal serum alpha-fetoprotein and normal ultrasonographic examinations. N Engl J Med 1990; 323:557-61.

3. Morrow RJ, McNay MB, Whittle MJ. Ultrasound detection of neural tube defects in patients with elevated maternal serum alpha-fetoprotein. Obstet Gynecol 1991; 78:1055-7.

4. Kollias SS, Goldstein RB, Cogen PH, Filly RA. Prenatally detected myelomeningoceles: sonographic accuracy in estimation of the spinal level. Radiology 1992; 185:109-12.

5. Cochrane DD, Wilson RD, Steinbok P, et al. Prenatal spinal evaluation and functional outcome of patients born with myelomeningocele: information for improved prenatal counselling and outcome prediction. Fetal Diagn Ther 1996; 11:159-68. 
6. Brumfield CG, Aronin PA, Cloud GA, Davis RO. Fetal myelomeningocele: is antenatal ultrasound useful in predicting neonatal outcome? J Reprod Med 1995; 40:26-30.

7. Biggio JR Jr, Owen J, Wenstrom KD, Oakes WJ. Can prenatal ultrasound findings predict ambulatory status in fetuses with open spina bifida? Am J Obstet Gynecol 2001; 185:1016-20.

8. Luthy DA, Wardinsky $\mathrm{T}$, Shurtleff DB, et al. Cesarean section before the onset of labor and subsequent motor function in infants with meningomyelocele diagnosed antenatally. N Engl J Med 1991; 324:662-6.

9. Shurtleff DB, Luthy DA, Nyberg RJ. Meningomyelocele: management in utero and pos natum. In: Bock G, Marsh J, editors. Neural Tube Defects. $1^{\text {st }}$ ed. London: John Wiley \& Sons; 1994. p.270-86. (CIBA Foundation Symposium, 181).
10. Coniglio SJ, Anderson SM, Ferguson JE $2^{\text {nd }}$. Functional motor outcome in children with myelomeningocele: correlation with anatomic level on prenatal ultrasound. Dev Med Child Neurol 1996; 38:675-80.

11.Thiagarajah S, Henke J, Hogge WA, Abbitt PL, Breeden N, Ferguson JE. Early diagnosis of spina bifida: the value of cranial ultrasound markers. Obstet Gynecol 1990; 76:54-7.

12.Nicolaides KH, Campbell S, Gabbe SG, Guidetti R. Ultrasound screening for spina bifida: cranial and cerebellar signs. Lancet 1986; 2:72-4.

13.Stark G, Drummond M. Spina bifida as an obstetric problem. Dev Med Child Neurol Suppl 1970; 22 Suppl 22:157.

14.Ralis ZA. Traumatizing effect of breech delivery on infants with spina bifida. J Pediatr 1975; 87:613-6.

Recebido em: 23/5/2003 Aceito com modificações em: 25/6/2003 